\title{
Holographic complexity for Lifshitz system
}

\author{
Sourav Karar ${ }^{1,2, *}$ and Sunandan Gangopadhyay ${ }^{2, \dagger}$ \\ ${ }^{1}$ Department of Physics, Government General Degree College, Muragachha 741154, Nadia, India \\ ${ }^{2}$ Department of Theoretical Sciences, S. N. Bose National Centre for Basic Sciences, Block-JD, Sector-III, \\ Salt Lake, Kolkata 700106, India
}

(Received 29 November 2017; revised manuscript received 26 June 2018; published 27 July 2018)

\begin{abstract}
The subregion holographic complexity of a $3+1$-dimensional Lifshitz spacetime having a scaling symmetry is computed. The change in the holographic complexity between the excited state and the ground state is then obtained. It is found that there is a nontrivial change in holographic complexity at first order in the perturbation of the pure Lifshitz geometry. The difference is next related to the changes in the energy and the entanglement chemical potential of the system. The calculation is carried out for both the values of the dynamical scaling exponent $z$ in the Lifshitz spacetime. The relations have a very similar form to the corresponding relation involving the change in entanglement entropy known to be an analogous relation to the first law of thermodynamics.
\end{abstract}

DOI: $10.1103 /$ PhysRevD.98.026029

\section{INTRODUCTION}

Information theory has made a profound contribution in understanding the laws of physics, namely, the thermodynamic derivation of Einstein's equations of general relativity [1] and the black hole information paradox [2]. Since entanglement and complexity are fundamental quantities in the theory of information, it is expected that they would play an important role in providing us a deeper understanding of the connection between the general theory of relativity and information theory.

Quantum entanglement [3-9] between two subsystems $A$ and $B$ for a given pure state can be characterized by a quantity called entanglement entropy (EE) and has been an important topic of intense research in the field of quantum information. It has also been realized that quantum information may be a useful tool to study black hole physics. The AdS/CFT correspondence, on the other hand, has been one of the most remarkable theoretical developments in providing deep insights in the realm of quantum gravity, black hole physics and strongly coupled condensed matter systems. This has led to the idea of constructing holographic gravitational descriptions of quantities relevant in the field of quantum information.

\footnotetext{
*sourav.karar91@gmail.com

† sunandan.gangopadhyay@gmail.com, sunandan.gangopadhyay@bose.res.in

Published by the American Physical Society under the terms of the Creative Commons Attribution 4.0 International license. Further distribution of this work must maintain attribution to the author(s) and the published article's title, journal citation, and DOI. Funded by SCOAP ${ }^{3}$.
}

A holographic description of quantum entanglement known as holographic EE [10,11] has been an important line of recent activity and has proven useful in computing the EE of conformal field theories [6-8]. The usefulness of $\mathrm{EE}$ is also manifest in describing systems away from equilibrium. In this context, an important question that has been asked in the literature [12] is whether an analogous relation to the first law of thermodynamics holds good in this case also. The answer to this question was found in the affirmative [12]. The change in EE was found to be proportional to the change in the energy of the system for sufficiently small subsystems. The constant of proportionality was found to be related to the size of the entangling region and was identified with the inverse of some temperature, called the entanglement temperature. The resemblance of the change in EE with the first law of thermodynamics was also confirmed in [13], where they computed the change in EE between an excited state of an AdS spacetime and pure AdS spacetime. However, the analyses in these cases were restricted to the relativistic systems. In [14], the question was addressed in the case of a nonrelativistic system, the well-known Lifshitz system in $3+1$ dimensions. The excited state that was considered there was nonisotropic. This resulted in the addition of an extra term in the change in entropy and was identified with an entanglement chemical potential.

Quantum complexity is another quantity in information theory which has been proved its importance in understanding the properties of horizons of black holes. The quantity intuitively indicate how difficult it is to map a system in a given state to another state. Motivated from studies in the holographic description of EE, investigations have been initiated in providing a holographic dual description of 
quantum complexity using the AdS/CFT correspondence. A prescription to compute the holographic complexity (HC) was proposed in $[15,16]$. The prescription tells that for subsystem $A$ in the boundary, if $V(\gamma)$ denotes the volume enclosed by the minimal hypersurface in the bulk, then the holographic subregion complexity is given by

$$
C_{V}=\frac{V(\gamma)}{8 \pi R G}
$$

where $R$ is the radius of curvature of the spacetime. It is to be noted that this prescription for computing complexity by evaluating the volume enclosed by the minimal hypersurface yields the subregion HC. In case of an asymptotically AdS spacetime, $R$ would be the AdS radius. This quantity was computed in $\mathrm{AdS}_{d+2}$ geometry in $[17,18]$.

There is yet another proposal for computing the $\mathrm{HC}$ of a system. According to this proposal, the $\mathrm{HC}$ of a system can be obtained from the bulk action evaluated on the WheelerDeWitt patch $[19,20]$,

$$
C_{W}=\frac{A(W)}{\pi \hbar},
$$

where $A(W)$ is the action evaluated on the Wheeler-DeWitt patch $W$ with a suitable boundary time. This quantity is called HC from the action.

In this paper, we shall compute the subregion $\mathrm{HC}$ for the nonrelativistic Lifshitz system in $3+1$ dimensions. We shall first compute it for the pure Lifshitz spacetime and then for the excited state. This would give us the change in the $\mathrm{HC}$ between the perturbed and the pure Lifshitz spacetimes,

$$
\Delta C_{V}=C\left(\gamma_{E L S}\right)-C\left(\gamma_{L S}\right),
$$

where $\gamma_{L S}$ is the minimal hypersurface for the pure Lifshitz spacetime and $\gamma_{E L S}$ is the minimal hypersurface for the excited Lifshitz geometry. The difference in $\mathrm{HC}$ is physically more relevant since both $C\left(\gamma_{L S}\right)$ and $C\left(\gamma_{E L S}\right)$ are divergent quantities; however, their difference is a finite quantity. We would then try to relate this change with changes in the energy, EE and entanglement chemical potential. This, in itself, is an interesting study since this would reveal the relation between complexity and EE. Similar investigation in trying to look at such a connection has been carried out in [21] for the near-horizon geometry of the D3-brane shell. Further, we shall carry out the analysis for both the values of the dynamical scaling exponent $z$ appearing in the Lifshitz spacetime.

The paper is organized as follows. In the next section, we compute the change in the $\mathrm{HC}$ between an excited state of the Lifshitz spacetime and the ground state. We then relate it to the components of the holographic stress tensor. We conclude in Sec. III.

\section{HOLOGRAPHIC COMPLEXITY FOR LIFSHITZ SPACETIME}

In this section, we will first compute the $\mathrm{HC}$, that is the Ryu-Takayanagi (RT) volume with a strip geometry for the unperturbed Lifshitz spacetime. We shall then compute the HC for the perturbed Lifshitz spacetime which corresponds to the excited state. This, in turn, would lead to the change in the $\mathrm{HC}$ between the excited and the unperturbed Lifshitz spacetimes. To begin with we shall present a brief description of the setup needed to compute the holographic complexity for the Lifshitz spacetime. The four-dimensional Lifshitz metric reads [22]

$$
\begin{gathered}
d s^{2}=-r^{2 z} d t^{2}+r^{2}\left(d x^{2}+d y^{2}\right)+\frac{d r^{2}}{r^{2}} \\
A=\alpha r^{z} d t, \quad \alpha^{2}=\frac{2(z-1)}{z} .
\end{gathered}
$$

The spacetime is the gravitational dual of a $2+1$ dimensional quantum many-body system with a Lifshitz symmetry near its quantum critical point. This solution can be obtained from the equations of motion following from the action $[23,24]$

$S=\frac{1}{16 \pi G_{4}} \int d^{4} x \sqrt{-g}\left(R-2 \Lambda-\frac{1}{4} F_{\mu \nu} F^{\mu \nu}-\frac{1}{2} m^{2} A_{\mu} A^{\mu}\right)$

with the choice $\Lambda=-\frac{1}{2}\left(z^{2}+z+4\right)$ for the cosmological constant and $m^{2}=2 z, A_{\mu}$ being a massive gauge field. It is evident that the above metric has the scaling symmetry $t \rightarrow \lambda^{z} t, x \rightarrow \lambda x, y \rightarrow \lambda y, r \rightarrow \lambda^{-1} r$, where $z$ is the dynamical scaling exponent.

The entangling region in the boundary is taken to be a straight belt with width $\ell$ such that $-\frac{\ell}{2} \leq x \leq \frac{\ell}{2}$ and $0 \leq y \leq L$, where $L$ is the extent of the subsystem in the other spatial direction. Since the strip has translational invariance along the direction $y$, one can describe the profile of the extremal surface by $x=x(r)$. With this setup in place, we can now proceed to compute the RT volume enclosed by the minimal surface extending from the boundary into the bulk. In this case, it is given by

$$
V^{(0)}=2 L \int_{r_{t}^{(0)}}^{\infty} d r r x(r),
$$

where $r_{t}^{(0)}$ is the turning point at which $r^{\prime}(x)=0$. To obtain the minimal surface $x(r)$, we write down the RT area functional considering $r=r(x)$. This is given by $[10,11]$

$$
\begin{aligned}
A^{(0)} & =\int_{-\frac{\ell}{2}}^{\frac{\ell}{2}} d x \int_{0}^{L} d y \sqrt{r^{\prime}(x)^{2}+r^{4}} \\
& =2 L \int_{0}^{\ell / 2} d x r^{2} \sqrt{1+\frac{r^{\prime}(x)^{2}}{r^{4}}},
\end{aligned}
$$

where / denotes derivative with respect to $x$. The minimization of this area functional determines the function $r^{\prime}(x)$ which reads 


$$
r^{\prime}(x)=\frac{r^{4}}{r_{t}^{(0) 2}} \sqrt{1-\frac{r_{t}^{(0) 4}}{r^{4}}} .
$$

This now leads to

$$
x(r)=\int_{r_{t}^{(0)}}^{r} d u \frac{r_{t}^{(0) 2}}{u^{4}} \frac{1}{\sqrt{1-\frac{r_{t}^{(0) 4}}{u^{4}}}} .
$$

Substituting the above expression for $x(r)$ in Eq. (6) and putting a cutoff $\delta$ for the $r$ integral, we have

$$
\begin{aligned}
V^{(0)} & =2 L \int_{r_{t}^{(0)}}^{\delta} d r r \int_{r_{t}^{(0)}}^{r} d u \frac{r_{t}^{(0) 2}}{u^{4}} \frac{1}{\sqrt{1-\frac{r_{t}^{(0) 4}}{u^{4}}}} \\
& =\frac{\sqrt{\pi} \Gamma\left(\frac{3}{4}\right) L \delta^{2}}{\Gamma\left(\frac{1}{4}\right) r_{t}^{(0)}}-\frac{\sqrt{\pi} \Gamma\left(\frac{5}{4}\right) L r_{t}^{(0)}}{\Gamma\left(\frac{3}{4}\right)}
\end{aligned}
$$

where we have ignored terms of order $1 / \delta$ since they are small.

Substituting Eq. (8) in Eq. (7) and once again using the cutoff $\delta$ for the $r$ integral and ignoring terms of order $1 / \delta$, we get

$$
\begin{aligned}
A^{(0)} & =2 L \int_{r_{t}^{(0)}}^{\delta} d r \frac{\left(\frac{r}{r_{t}^{(0)}}\right)^{2}}{\sqrt{\left(\frac{r}{r_{t}^{(0)}}\right)^{4}-1}} \\
& =2 L \delta-\frac{5}{3} L r_{t}^{(0)} .
\end{aligned}
$$

From Eq. (8), we also have

$$
\frac{\ell}{2}=\int_{0}^{\frac{\ell}{2}} d x=\int_{r_{t}^{(0)}}^{\infty} d r \frac{1}{r^{2} \sqrt{\left(\frac{r}{r_{t}^{(0)}}\right)^{4}-1}}=\frac{\sqrt{\pi} \Gamma\left(\frac{3}{4}\right)}{\Gamma\left(\frac{1}{4}\right) r_{t}^{(0)}} .
$$

We know that the $\mathrm{HC}$ is proportional to the volume enclosed by the minimum surface extending from the boundary into the bulk, which in this case is given by

$$
\begin{aligned}
C_{V}^{(0)} & =\frac{V^{(0)}}{8 \pi G_{4}} \\
& =\frac{\sqrt{\pi}}{8 \pi G_{4}}\left[\frac{\Gamma\left(\frac{3}{4}\right) L \delta^{2}}{\Gamma\left(\frac{1}{4}\right) r_{t}^{(0)}}-\frac{\Gamma\left(\frac{5}{4}\right) L r_{t}^{(0)}}{\Gamma\left(\frac{3}{4}\right)}\right] .
\end{aligned}
$$

This is the HC for the ground state of the Lifshitz spacetime. It should be noted that the calculation of the $\mathrm{HC}$ is in a subregion and is usually referred to as subregion $\mathrm{HC}$ in the literature [25].

We shall now proceed to calculate the $\mathrm{HC}$ for the perturbed Lifshitz metric [22]

$$
\begin{aligned}
d s^{2}= & -r^{2 z}\left(1+h_{t t}(r)\right) d t^{2}+r^{2}\left(1+h_{x x}(r)\right) d x^{2} \\
& +r^{2}\left(1+h_{y y}(r)\right) d y^{2}+\frac{d r^{2}}{r^{2}}+2\left[-r^{2 z} v_{1 x}(r)\right. \\
& \left.+r^{2} v_{2 x}(r)\right] d t d x+2\left[-r^{2 z} v_{1 y}(r)+r^{2} v_{2 y}(r)\right] d t d y \\
& +2 r^{2} h_{x y}(r) d x d y \\
A & =\alpha r^{2}\left[\left(1+a_{t}(r)+\frac{1}{2} h_{t t}(r)\right) d t+v_{1 x}(r) d x+v_{1 y}(r) d y\right] .
\end{aligned}
$$

This can be considered as the excited state of the Lifshitz spacetime. An asymptotically Lifshitz spacetime results when $h_{t t}(r), h_{x x}(r), h_{y y}(r), v_{1 x}(r), v_{2 x}(r), v_{1 y}(r), v_{2 y}(r)$, $h_{x y}(r)$, and $a_{t}(r) \rightarrow 0$ as $r \rightarrow \infty$. One can now define

$$
\begin{aligned}
h_{t t}(r) & =f(r) \\
h_{x x}(r) & =k(r)+t_{d}(r) \\
h_{y y}(r) & =k(r)-t_{d}(r) \\
a_{t} & =j(r) .
\end{aligned}
$$

Substituting this in Eq. (5), the linearized action can be obtained [22]. The solutions obtained by solving the equations of motion (in the radial gauge) resulting from the linearized action have the form $[22,26,27]$ for $z=2$

$$
\begin{aligned}
j(r) & =-\frac{c_{1}+c_{2} \ln r}{r^{4}}, \\
f(r) & =\frac{4 c_{1}-5 c_{2}+4 c_{2} \ln r}{12 r^{4}}, \\
k(r) & =\frac{4 c_{1}+5 c_{2}+4 c_{2} \ln r}{24 r^{4}}, \\
t_{d}(r) & =\frac{t_{d 2}}{r^{4}}
\end{aligned}
$$

and for $z \neq 2$

$$
\begin{aligned}
& j(r)=-\frac{(z+1) c_{1}}{(z-1) r^{z+2}}-\frac{(z+1) c_{2}}{(z-1) r^{\frac{1}{2}\left(z+2+\beta_{z}\right)},} \\
& f(r)=4 \frac{1}{(z+2)} \frac{c_{1}}{r^{z+2}}+2 \frac{\left(5 z-2-\beta_{z}\right)}{\left(z+2+\beta_{z}\right)} \frac{c_{2}}{r^{\frac{1}{2}\left(z+2+\beta_{z}\right)}}, \\
& k(r)=2 \frac{1}{(z+2)} \frac{c_{1}}{r^{z+2}}-2 \frac{\left(3 z-4-\beta_{z}\right)}{\left(z+2+\beta_{z}\right)} \frac{c_{2}}{r^{\frac{1}{2}\left(z+2+\beta_{z}\right)}}, \\
& t_{d}(r)=\frac{t_{d 2}}{r^{z+2}}
\end{aligned}
$$

where $\beta_{z}^{2}=9 z^{2}-20 z+20=(z+2)^{2}+8(z-1)(z-2)$, $c_{1}, c_{2}$ and $t_{d 2}$ are constants of integration.

With this setup in place, we can now move on to compute the $\mathrm{HC}$ for the perturbed Lifshitz metric (14). This in turn would lead to the change in complexity due to the change in the metric. In the computation of the complexity of the excited state, we shall keep the length of the entangling region $\ell$ fixed. Now as the entangling 
region is same but the metric is perturbed, hence the turning point changes. Let $r_{t}$ be the new turning point such that $r_{t}=r_{t}^{(0)}+\delta r_{t}$, where $\delta r_{t}$ is the change in the turning point.

As in the unperturbed case, we start by considering the minimal surface to be parametrized by $x=x(r)$. The volume in the part of the bulk geometry enclosed by the minimal surface is given by

$V=\int_{-\frac{\ell}{2}}^{\frac{\ell}{2}} d x \int_{0}^{L} d y \int_{r_{t}}^{\delta} d r r x(r) \sqrt{1+h_{x x}(r)+h_{y y}(r)}$.

To determine $x(r)$, we once again start by writing down the area as a functional of the minimal surface $r=r(x)$. This reads

$$
A=2 L \int_{0}^{\ell / 2} d x \sqrt{r^{\prime 2}(x)\left[1+h_{y y}(r)\right]+r^{4}\left[1+h_{x x}(r)+h_{y y}(r)\right]} .
$$

Regarding $x$ as a time, one can easily obtain the Hamiltonian which does not depend on $x$. This in turn leads to

$$
r^{\prime}(x)=\frac{\sqrt{\frac{r^{8}}{Q}\left[1+h_{x x}(r)+h_{y y}(r)\right]^{2}-r^{4}\left[1+h_{x x}(r)+h_{y y}(r)\right]}}{\sqrt{1+h_{y y}(r)}}
$$

where $Q$ is a constant of integration. This gets fixed by the fact that $r^{\prime}(x)=0$ at the turning point $r=r_{t}$. Hence we have

$$
Q=r_{t}^{4}\left[1+h_{x x}\left(r_{t}\right)+h_{y y}\left(r_{t}\right)\right] .
$$

Equation (20) determines the minimal surface $r=r(x)$ or $x=x(r)$.

Integrating Eq. (20) from $x=0$ to $x=\ell / 2$ assuming that the $h$ 's are small, ${ }^{1}$ we obtain upto first order in $h$

$$
\begin{aligned}
\frac{\ell}{2}= & \int_{r_{t}}^{\infty} \frac{d r}{r^{2} f\left(r, r_{t}\right)}\left\{1-h_{x x}(r)-\frac{1}{2} h_{y y}(r)\right. \\
& +\frac{1}{2}\left[h_{x x}\left(r_{t}\right)+h_{y y}\left(r_{t}\right)\right] \\
& \left.+\frac{\left[h_{x x}\left(r_{t}\right)+h_{y y}\left(r_{t}\right)-h_{x x}(r)-h_{y y}(r)\right]}{2 f^{2}\left(r, r_{t}\right)}\right\}
\end{aligned}
$$

where $f^{2}\left(r, r_{t}\right)=\left(r / r_{t}\right)^{4}-1$. Now since we keep $\ell$ fixed, therefore we also have

\footnotetext{
${ }^{1}$ Note that the length of the strip $(\ell)$ is assumed to be small so that the hypersurface in the bulk can penetrate only the ultraviolet region of the perturbed Lifshitz spacetime. Holographically this implies that only the asymptotic behaviour of the perturbed Lifshitz spacetime [Eq. (14)] needs to be considered.
}

$$
\frac{\ell}{2}=\int_{r_{t}^{(0)}}^{\infty} \frac{d r}{r^{2} f\left(r, r_{t}^{(0)}\right)} .
$$

Equating the above two expressions gives

$$
\begin{aligned}
\delta r_{t}= & -N r_{t}^{(0)} r_{t} \int_{r_{t}}^{\infty} \frac{d r}{r^{2} f\left(r, r_{t}\right)}\left\{h_{x x}(r)+\frac{1}{2} h_{y y}(r)\right. \\
& -\frac{1}{2}\left[h_{x x}\left(r_{t}\right)+h_{y y}\left(r_{t}\right)\right] \\
& \left.-\frac{\left[h_{x x}\left(r_{t}\right)+h_{y y}\left(r_{t}\right)-h_{x x}(r)-h_{y y}(r)\right]}{2 f^{2}\left(r, r_{t}\right)}\right\}
\end{aligned}
$$

where

$$
N=\left\{\int_{1}^{\infty} \frac{d \xi}{\xi^{2} \sqrt{\xi^{4}-1}}\right\}^{-1}=\frac{\Gamma(1 / 4)}{\sqrt{\pi} \Gamma(3 / 4)}
$$

Requiring $\delta r_{t}=0$ [13] leads to the following condition on the perturbations:

$$
\begin{aligned}
\frac{\left[h_{x x}\left(r_{t}\right)+h_{y y}\left(r_{t}\right)\right]}{2 f^{2}\left(r, r_{t}\right)}= & \frac{1}{\left[1+f^{2}\left(r, r_{t}\right)\right]}\left\{h_{x x}(r)\left(1+\frac{1}{2 f^{2}\left(r, r_{t}\right)}\right)\right. \\
& \left.+\frac{h_{y y}(r)}{2}\left(1+\frac{1}{f^{2}\left(r, r_{t}\right)}\right)\right\} .
\end{aligned}
$$

Further, since $\delta r_{t}=0$, we have $r_{t}=r_{t}^{(0)}$ in all the equations. Using this condition in Eqs. (19) and (20) yields

$$
A=A^{(0)}+L \int_{r_{t}^{(0)}}^{\delta} d r \frac{\left[h_{y y}(r)+\left(\frac{r_{t}^{(0)}}{r}\right)^{4} h_{x x}(r)\right]}{\sqrt{1-\left(\frac{r_{t}^{(0)}}{r}\right)^{4}}}
$$

and the same expression for $x(r)$ as in Eq. (9). Substituting the form of $x(r)$ [in Eq. (9)] in Eq. (18) and keeping terms up to first order in $h$, we obtain

$$
\begin{aligned}
V= & V^{(0)}+L \int_{r_{t}^{(0)}}^{\delta} d r r\left[h_{x x}(r)+h_{y y}(r)\right] \\
& \times \int_{r_{t}^{(0)}}^{r} d u \frac{r_{t}^{(0) 2}}{u^{4}} \frac{1}{\sqrt{1-\frac{r_{t}^{(0) 4}}{u^{4}}}} .
\end{aligned}
$$

Note that since we are considering small perturbations around the background spacetime, $h(r)$ 's are small. ${ }^{2}$ This in turn implies that the turning point $r_{t}$ is close to the boundary. However, since $r=\infty$ at the boundary and $\ell$ is inversely related to $r_{t}$, hence $\ell$ is small.

The change in HC due to the perturbation of the Lifshitz spacetime is given by

\footnotetext{
${ }^{2}$ Note that the smallness of $h(r)$ 's is related to the smallness of the system length $(\ell)$ in the boundary.
} 


$$
\begin{aligned}
\Delta C_{V}= & \frac{\Delta V}{8 \pi G_{4}} \\
= & \frac{L}{8 \pi G_{4}} \int_{r_{t}^{(0)}}^{\delta} d r r\left[h_{x x}(r)+h_{y y}(r)\right] \\
& \times \int_{r_{t}^{(0)}}^{r} d u \frac{r_{t}^{(0) 2}}{u^{4}} \frac{1}{\sqrt{1-\frac{r_{t}^{(0) 4}}{u^{4}}}} .
\end{aligned}
$$

Now we make use of the expressions for the perturbations to compute the $\mathrm{HC}$ explicitly. For $z=2$, substituting the expressions for $h_{x x}(r)$ and $h_{y y}(r)$ from Eqs. (15) and (16), we obtain

$\Delta C_{V}=\frac{\sqrt{\pi} \Gamma\left(\frac{5}{4}\right) L}{8 \pi G_{4} \Gamma\left(\frac{7}{4}\right) r_{t}^{(0) 3}}\left(\frac{c_{1}+c_{2} \ln r_{t}^{(0)}}{24}+\frac{3 \pi+13}{288} c_{2}\right)$.

A few observations are in order at this point. We would first like to note that a similar relation for the change in holographic EE was obtained in [14]. However, the expression contained a term involving $t_{d 2}$ which is absent in the above expression for the difference in HC. Further, the above change in $\mathrm{HC}$ is finite although both $V$ and $V^{(0)}$ [Eqs. (10) and (28)] are divergent quantities. It has been pointed out in [28] that the finiteness of the change in $\mathrm{HC}$ is crucial in proposing the holographic dual to Fisher information metric which reads $[29,30]$

$G_{F, \lambda_{i} \lambda_{j}}(\lambda)=\int_{x} p(x, \lambda) \frac{\partial}{\partial \lambda_{i}} \log p(x, \lambda) \frac{\partial}{\partial \lambda_{j}} \log p(x, \lambda) d x$

where $\lambda=\left(\lambda_{1}, \ldots, \lambda_{n}\right)$ are the parameters characterizing a pure state $\left|\psi_{\lambda_{i}}\right\rangle$ of a quantum mechanical system, $n$ denotes the dimension of the statistical manifold and $p(x, \lambda)$ 's can be identified as the pure state density matrices. The proposal for the holographic dual to the Fisher information metric was suggested in [28] and reads

$$
G_{F, m m}(\lambda)=\partial_{m}^{2} \mathcal{F}
$$

where $\mathcal{F} \sim \Delta C_{V}$ (upto prefactors) and $m$ is the perturbation parameter in the bulk geometry considered by them.

We would next like to point that the change in the HC between the excited state of the Lifshitz spacetime and the pure Lifshitz geometry occurs at first order in the perturbation $h_{x x}(r)$ and $h_{y y}(r)$. This is in contrast to the observation made in [17] where there is no change in the HC between the excited AdS geometry and pure AdS geometry at first order in the perturbation and the change occurs at second order. The above expression for the change in $\mathrm{HC}$ is important because it can be related to the change in holographic EE as we shall see in the subsequent discussion. Further, we shall also find that the change in $\mathrm{HC}$ can be related to the change in energy and hence the relation can be regarded as an analogous expression corresponding to the first law of entanglement thermodynamics.

Our next step is to express the change in $\mathrm{HC}\left(\Delta C_{V}\right)$ in terms of the holographic energy-momentum tensor. The motivation of doing this is to obtain a relation between the change in $\mathrm{HC}$ and the change in energy which in turn would lead to a relation between the change in $\mathrm{HC}$ and the change in holographic EE. The various components of the stress tensor can be found by varying the action with respect to the boundary metric. The expressions for the energy and pressure densities in terms of the functions defined earlier read [22]

$$
\begin{aligned}
& T_{t t}=-r^{z+2}\left[2 r \partial_{r} k(r)+\alpha^{2}\left(z j(r)+r \partial_{r} j(r)+\frac{1}{2} r \partial_{r} f(r)\right)\right] \\
& T_{x x}=-2 r^{z+2}\left[(z-1) j(r)-\frac{r}{2} \partial_{r} f(r)-\frac{r}{2} \partial_{r} k(r)-\frac{1}{2}(z+2) t_{d}(r)\right] \\
& T_{y y}=-2 r^{z+2}\left[(z-1) j(r)-\frac{r}{2} \partial_{r} f(r)-\frac{r}{2} \partial_{r} k(r)+\frac{1}{2}(z+2) t_{d}(r)\right] .
\end{aligned}
$$

It should be noted that the above expressions for the various components of the stress tensor is valid for the vacuum solution. The addition of a scalar field of mass $m$ to the gravity action (5) is interesting in its own right and has been considered in [31]. It has been pointed out there that the finiteness of the scalar field action in Lifshitz spacetime requires a $z$-dependent BreitenlohnerFreedman bound [32,33]. However, a thorough analysis of the stress tensor in the presence of a scalar field coupled to the Lifshitz spacetime has been missing in the literature. Hence investigating what happens if matter is added to the background geometry would require an in depth analysis and therefore would warrant a separate investigation by itself. The results that follow in the subsequent discussion are indeed valid for the vacuum solution.

Substituting the forms of the functions for $z=2$ [given in Eq. (16)] in the above expressions [14], we have 


$$
\begin{gathered}
\left\langle T_{x x}\right\rangle=\frac{1}{16 \pi G_{4}}\left(\frac{4 c_{2}}{3}+4 t_{d 2}\right) \\
\left\langle T_{t t}\right\rangle=\frac{1}{16 \pi G_{4}} \frac{4 c_{2}}{3} .
\end{gathered}
$$

Substituting $c_{2}$ from Eq. (35) in Eq. (30), we obtain

$$
\Delta C_{V}=\frac{\sqrt{\pi} \Gamma\left(\frac{5}{4}\right) L}{192 \Gamma\left(\frac{7}{4}\right) r_{t}^{(0) 3}}\left(\frac{c_{1}+c_{2} \ln r_{t}^{(0)}}{\pi G_{4}}+(3 \pi+13)\left\langle T_{t t}\right\rangle\right) .
$$

Now using the fact that the total energy, entanglement pressure, entanglement chemical potential of the excited state and charge are given by [14]

$$
\begin{gathered}
\Delta E=\int_{0}^{L} d y \int_{-\ell / 2}^{\ell / 2} d x\left\langle T_{t t}\right\rangle=L \ell\left\langle T_{t t}\right\rangle \\
\Delta P_{x}=\left\langle T_{x x}\right\rangle \\
\Delta \mu=\frac{1}{12 \pi G_{4}}\left(c_{1}+c_{2} \ln r_{t}^{(0)}\right) \\
Q=m^{2} \alpha L \ell=4 L \ell ; \quad \text { for } z=2
\end{gathered}
$$

the above expression for the change in the $\mathrm{HC}$ can be recast as

$$
\Delta C_{V}=\frac{1}{T_{\mathrm{ent}}}\left[B_{1} \Delta \mu Q+B_{2} \Delta E\right]
$$

with

$$
B_{1}=\frac{25 \Gamma\left(\frac{5}{4}\right) \Gamma\left(\frac{1}{4}\right)}{32 \pi(54-5 \pi) \Gamma\left(\frac{7}{4}\right) \Gamma\left(\frac{3}{4}\right)}, \quad B_{2}=\left(\frac{3 \pi+13}{3}\right) B_{1}
$$

and $T_{\text {ent }}$ is the entanglement temperature

$$
T_{\mathrm{ent}}=\frac{24 r_{t}^{2}}{\pi} \frac{25}{(324-30 \pi)}=\frac{96 \Gamma^{2}\left(\frac{3}{4}\right)}{\ell^{2} \Gamma^{2}\left(\frac{1}{4}\right)} \frac{25}{(324-30 \pi)}
$$

appearing in the expression for the first law of entanglement thermodynamics for an excited state of the Lifshitz spacetime [14]

$\Delta E=T_{\mathrm{ent}} \Delta S+\frac{10}{(54-5 \pi)} \Delta P_{x} V-\frac{5}{(54-5 \pi)} \Delta \mu Q$.

Equation (41) relates the change in the $\mathrm{HC}$ with the change in energy and the change in entanglement chemical potential. It is to be noted that the change in pressure $\Delta P_{x}$ does not appear in the expression for the change in $\mathrm{HC}$ in contrast to the expression for the change in the holographic EE. Note that the origin of $\Delta P_{x}$ in the change in $\operatorname{EE}(\Delta S)$ is due to the presence of the $t_{d 2}$ term (related to $\Delta P_{x}$ ) in $\Delta S$ [14] which arises from the combination $h_{y y}(r)+\left(\frac{r_{t}}{r}\right)^{4} h_{x x}(r)$. However in the case of $\Delta C_{V}$, the combination $h_{x x}(r)+h_{y y}(r)$ arises which results in the cancellation of the $t_{d 2}$ term responsible for the origin of $\Delta P_{x}$.

We can recast Eq. (41) in another form. Rewriting Eq. (41) as

$$
\Delta E=\frac{1}{B_{1}} T_{\mathrm{ent}} \Delta C_{V}-\frac{B_{2}}{B_{1}} \Delta \mu Q
$$

and equating this with Eq. (44), we have

$\Delta C_{V}=B_{1} \Delta S+\frac{10 B_{1}}{54-5 \pi} \frac{\Delta P_{x} V}{T_{\mathrm{ent}}}+\left(B_{2}-\frac{5 B_{1}}{54-5 \pi}\right) \frac{\Delta \mu Q}{T_{\mathrm{ent}}}$.

This expression relates the change in the $\mathrm{HC}$ with the change in holographic EE, change in pressure and the change in entanglement chemical potential.

We now compute the change in the HC from Eq. (29) for $z \neq 2$. For this, we substitute the expressions for $h_{x x}(r)$ and $h_{y y}(r)$ from Eqs. (15) and (17) in Eq. (29). The change is

$$
\begin{aligned}
\Delta C_{V}= & \frac{\sqrt{\pi} L}{8 \pi G_{4} r_{t}^{(0) z+1}}\left[\frac{c_{1} \Gamma\left(\frac{z+3}{4}\right)}{z(z+2) \Gamma\left(\frac{z+5}{4}\right)}\right. \\
& \left.+\frac{2 c_{2}\left(4+\beta_{z}-3 z\right) \Gamma\left(\frac{z+\beta_{z}+4}{8}\right)}{\left(z+\beta_{z}+2\right)\left(z+\beta_{z}-2\right) \Gamma\left(\frac{z+\beta_{z}+8}{8}\right)}\right] .
\end{aligned}
$$

The energy-momentum tensor and the entanglement chemical potential for $z \neq 2$ can be obtained from Eq. (33) using Eq. (17), and they read

$$
\left\langle T_{t t}\right\rangle=\frac{1}{4 \pi G_{4}}\left(\frac{z-2}{z}\right) c_{1}
$$

$\Delta \mu=\frac{1}{16 \pi G_{4}} \frac{\alpha}{[z-1]}\left[4 c_{1}+c_{2} z\left(4+\beta_{z}-3 z\right) r_{t}^{(0) \frac{1}{2}\left(z+2-\beta_{z}\right)}\right]$.

We now invert the above expressions to obtain $c_{1}$ and $c_{2}$ as functions of $\left\langle T_{t t}\right\rangle$ and $\Delta \mu$. These read

$$
\begin{aligned}
c_{1} & =4 \pi G_{4} \frac{z}{z-2}\left\langle T_{t t}\right\rangle \\
c_{2} & =\frac{16 \pi G_{4}\left[(z-1)(z-2) \Delta \mu-\alpha z\left\langle T_{t t}\right\rangle\right]}{\alpha z(z-2)\left(4+\beta_{z}-3 z\right) r_{t}^{\frac{1}{2}\left(z+2-\beta_{z}\right)}} .
\end{aligned}
$$

Now the entanglement temperature appearing in the first law of entanglement thermodynamics for Lifshitz system with $z \neq 2$ reads [14] 


$$
T_{\mathrm{ent}}=\frac{2 \Gamma\left(\frac{3}{4}\right) r_{t}^{(0) z}}{\pi \Gamma\left(\frac{1}{4}\right) K_{1}}
$$

where

$$
\begin{aligned}
K_{1}= & \frac{z^{2}}{(z+3)\left(z^{2}-4\right)} \frac{\Gamma\left(\frac{1+z}{4}\right)}{\Gamma\left(\frac{3+z}{4}\right)} \\
& -\frac{2}{(z-2)\left(4+z+\beta_{z}\right)} \frac{\Gamma\left(\frac{z+\beta_{z}}{8}\right)}{\Gamma\left(\frac{z+4+\beta_{z}}{8}\right)} .
\end{aligned}
$$

Using the expressions for $c_{1}$ and $c_{2}$ from Eq. (50) and using Eq. (51), we can write Eq. (47) as

$$
\Delta C_{V}=\frac{1}{T_{\mathrm{ent}}}\left[D_{1} \Delta E+D_{2} \Delta \mu Q\right],
$$

where $D_{1}$ and $D_{2}$ are given by

$$
\begin{aligned}
D_{1}= & \frac{2}{\pi K_{1}}\left[\frac{\Gamma\left(\frac{z+3}{4}\right)}{4\left(z^{2}-4\right) \Gamma\left(\frac{z+5}{4}\right)}\right. \\
& \left.-\frac{2}{(z-2)\left(z+\beta_{z}+2\right)\left(z+\beta_{z}-2\right)} \frac{\Gamma\left(\frac{z+\beta_{z}+4}{8}\right)}{\Gamma\left(\frac{z+\beta_{z}+8}{8}\right)}\right] \\
D_{2}= & \frac{1}{\pi z\left(z+\beta_{z}+2\right)\left(z+\beta_{z}-2\right) K_{1}} \frac{\Gamma\left(\frac{z+\beta_{z}+4}{8}\right)}{\Gamma\left(\frac{z+\beta_{z}+8}{8}\right)}
\end{aligned}
$$

and we have used the fact that $Q=m^{2} \alpha L \ell=\sqrt{8 z(z-1)}$ is the total charge. Once again, we find that the change in $\mathrm{HC}$ gets related to the change in energy and the change in entanglement chemical potential.

Now the first law of entanglement thermodynamics for the Lifshitz system with $z \neq 2$ reads [14]

$$
\Delta E=T_{\mathrm{ent}} \Delta S+\frac{K_{2}}{K_{1}} \Delta P_{x} V-\frac{K_{3}}{K_{1}} \Delta \mu Q,
$$

where

$$
\begin{aligned}
& K_{2}=\frac{\Gamma\left(\frac{1+z}{4}\right)}{(z+2)(z+3) \Gamma\left(\frac{3+z}{4}\right)}, \\
& K_{3}=\frac{\Gamma\left(\frac{z+\beta_{z}}{8}\right)}{2 z\left(4+z+\beta_{z}\right) \Gamma\left(\frac{z+4+\beta_{z}}{8}\right)} .
\end{aligned}
$$

To relate the change in $\mathrm{HC}$ with the change in $\mathrm{EE}$, we once again rewrite Eq. (53) as

$$
\Delta E=\frac{1}{D_{1}} \Delta C_{V} T_{\text {ent }}-\frac{D_{2}}{D_{1}} \Delta \mu Q
$$

and equate with Eq. (55) to get

$$
\Delta C_{V}=D_{1} \Delta S+\frac{K_{2} D_{1}}{K_{1}} \frac{\Delta P_{x} V}{T_{e n t}}+\left(D_{2}-\frac{K_{3} D_{1}}{K_{1}}\right) \frac{\Delta \mu Q}{T_{\text {ent }}} .
$$

This expression once again relates the change in the $\mathrm{HC}$ with the change in holographic EE, the change in pressure and the change in entanglement chemical potential. The relation is the counterpart of the corresponding relation (46) for $z=2$. The expression of $K_{1}$ [Eq. (52)] appearing in the above relation for the change in holographic complexity is finite for values of $z$ lying in the range $1 \leq z<2$ and $z>2$.

\section{CONCLUSIONS}

In this paper, the holographic subregion complexity of a 3 + 1-dimensional Lifshitz spacetime has been computed. The same has been computed for the perturbed Lifshitz spacetime by considering subsystems with the entangling region sufficiently small in size. This has led to the result for the change in holographic subregion complexity between the excited state and the ground state of Lifshitz spacetime. We have observed that the change in the holographic subregion complexity between the excited state of the Lifshitz spacetime and the pure Lifshitz geometry occurs at first order in the perturbation $h_{x x}(r)$ and $h_{y y}(r)$. This is in contrast to the observation made in [17], where there is a change in the holographic complexity between the excited AdS geometry and pure AdS geometry only at second order in the perturbation. It should be further noted that the change in holographic complexity may be caused by the change of the turning point, the change of the solution for the minimal hypersurface and the change of the spacetime geometry. The interesting point is that the change of holographic complexity is completely given by the change of the metric at leading order. The analysis has been carried out for both the values of the dynamical scaling exponent $z$ appearing in the Lifshitz spacetime. The change in the holographic subregion complexity is then expressed in terms of the components of the holographic stress tensor, the motivation for which is to relate it to the change in the energy, change in holographic entanglement entropy and the entanglement chemical potential. This relation can be regarded as an analogous expression corresponding to the first law of entanglement thermodynamics. The finiteness of the expression for the change in holographic complexity is also important for proposing the holographic dual to Fisher information. It is also observed that the change in pressure does not arise in the expression for the change in the holographic complexity in contrast to the change in entanglement entropy.

The calculations carried out in this paper are for striplike subregions but the results would also have a similar form for spherical-disk-like subregions. This will happen due to 
the fact that the change in geometry of the subregion will only change the coefficients of various terms in the expressions for the change in holographic complexity; however, the form of the expressions will remain unchanged. We would also like to mention that we have carried out our computations (for the change in subregion holographic complexity) for constant perturbation (along the boundary directions) to the background geometry. It would indeed be very interesting to do the same analysis for general perturbations to the pure Lifshitz geometry. It would also be very instructive to extend our analysis for Lifshitz theories with hyperscaling violation and Lifshitz black holes. Finally, a thorough study of the stress tensor in the presence of a scalar field coupled to the gravity action would be necessary to investigate the status of the results obtained in this paper, for instance the relation between the change in holographic complexity to the change in holographic entanglement entropy. Work in this direction is in progress, and we hope to report this in the future.

\section{ACKNOWLEDGMENTS}

S. G. acknowledges the support by DST SERB under a Start Up Research Grant (Young Scientist), File No. YSS/ 2014/000180. S. G. would also like to thank Inter University Centre for Astronomy and Astrophysics (IUCAA), Pune, for the Visiting Associateship. The authors would like to thank the referee for very illuminating comments. S. K. and S. G. contributed equally to this work.
[1] T. Jacobson, Thermodynamics of Spacetime: The Einstein Equation of State, Phys. Rev. Lett. 75, 1260 (1995).

[2] S. W. Hawking, Particle creation by black holes, Commun. Math. Phys. 43, 199 (1975).

[3] L. Bombelli, R. K. Koul, J. Lee, and R. D. Sorkin, A quantum source of entropy for black holes, Phys. Rev. D 34, 373 (1986).

[4] M. Srednicki, Entropy and Area, Phys. Rev. Lett. 71, 666 (1993).

[5] C. Holzhey, F. Larsen, and F. Wilczek, Geometric and renormalized entropy in conformal field theory, Nucl. Phys. B424, 443 (1994).

[6] P. Calabrese and J. L. Cardy, Entanglement entropy and quantum field theory, J. Stat. Mech. (2004) P06002.

[7] P. Calabrese and J. L. Cardy, Entanglement entropy and quantum field theory: A Non-technical introduction, Int. J. Quantum. Inform. 04, 429 (2006).

[8] P. Calabrese and J. Cardy, Entanglement entropy and conformal field theory, J. Phys. A: Math. Theor. 42, 504005 (2009).

[9] J. Eisert, M. Cramer, and M. B. Plenio, Area laws for the entanglement entropy—a review, Rev. Mod. Phys. 82, 277 (2010).

[10] S. Ryu and T. Takayanagi, Holographic Derivation of Entanglement Entropy from AdS/CFT, Phys. Rev. Lett. 96, 181602 (2006).

[11] S. Ryu and T. Takayanagi, Aspects of holographic entanglement entropy, J. High Energy Phys. 08 (2006) 045.

[12] J. Bhattacharya, M. Nozaki, T. Takayanagi, and T. Ugajin, Thermodynamical Property of Entanglement Entropy for Excited States, Phys. Rev. Lett. 110, 091602 (2013).

[13] D. Allahbakhshi, M. Alishahiha, and A. Naseh, Entanglement thermodynamics, J. High Energy Phys. 08 (2013) 102.

[14] S. Chakraborty, P. Dey, S. Karar, and S. Roy, Entanglement thermodynamics for an excited state of Lifshitz system, J. High Energy Phys. 04 (2015) 133.
[15] L. Susskind, Computational complexity and black hole horizons, Fortschr. Phys. 64, 24 (2016); Addendum 64, 44(A) (2016).

[16] D. Stanford and L. Susskind, Complexity and shock wave geometries, Phys. Rev. D 90, 126007 (2014).

[17] M. Alishahiha, Holographic complexity, Phys. Rev. D 92, 126009 (2015).

[18] O. B. Amia and D. Carmia, On volumes of subregions in holography and complexity, J. High Energy Phys. 11 (2016) 129.

[19] A. R. Brown, D. A. Roberts, L. Susskind, B. Swingle, and Y. Zhao, Holographic Complexity Equals Bulk Action?, Phys. Rev. Lett. 116, 191301 (2016).

[20] A. R. Brown, D. A. Roberts, L. Susskind, B. Swingle, and Y. Zhao, Complexity, action and black holes, Phys. Rev. D 93, 086006 (2016).

[21] D. Momeni, M. Faizal, A. Myrzakul, S. Bahamonde, and R. Myrzakulov, A holographic bound for D3-brane, Eur. Phys. J. C, 77, 391 (2017).

[22] S. F. Ross and O. Saremi, Holographic stress tensor for non-relativistic theories, J. High Energy Phys. 09 (2009) 009.

[23] S. Kachru, X. Liu, and M. Mulligan, Gravity duals of Lifshitz-like fixed points, Phys. Rev. D 78, 106005 (2008).

[24] M. Taylor, Non-relativistic holography, arXiv:0812.0530.

[25] P. Roy and T. Sarkar, Note on subregion holographic complexity, Phys. Rev. D 96, 026022 (2017).

[26] S. F. Ross, Holography for asymptotically locally Lifshitz spacetimes, Classical Quantum Gravity 28, 215019 (2011).

[27] T. Andrade and S. F. Ross, Boundary conditions for metric fluctuations in Lifshitz, Classical Quantum Gravity 30, 195017 (2013).

[28] S. Banerjee, J. Erdmenger, and D. Sarkar, A holographic dual to Fisher information and its relation with bulk entanglement, Proc. Sci., CORFU2016 (2017) 092.

[29] M. A. Nielsen and I. L. Chuang, Quantum Computation and Quantum Information (Cambridge University Press, Cambridge, England, 2010). 
[30] D. Spehner, Quantum correlations and distinguishability of quantum states, J. Math. Phys. 55, 075211 (2014).

[31] A. Giacomini, G. Giribet, M. Leston, J. Oliva, and S. Ray, Scalar field perturbations in asymptotically Lifshitz black holes, Phys. Rev. D 85, 124001 (2012).
[32] P. Breitenlohner and D.Z. Freedman, Positive energy in anti-de Sitter backgrounds and gauged extended supergravity, Phys. Lett. B 115, 197 (1982).

[33] P. Breitenlohner and D. Z. Freedman, Stability in gauged extended supergravity, Ann. Phys. (N.Y.) 144, 249 (1982). 